\title{
Diagnostic assessment program for prostate cancer: Lessons learned after 2 years and degree of compliance to Canadian guidelines
}

\author{
Waleed Shabana, Ahmed Kotb, Daniel Tesolin, Mohammed F.K. Ibrahim, Kristi Dolcetti, Amy Boucher, \\ Mohammed Bassuony, Kevin Ramchandar, Ahmed S. Zakaria, Hazem Elmansy, Walid Shahrour \\ Northern Ontario School of Medicine, Thunder Bay, Ontario, Canada.
}

\begin{abstract}
Summary Background: In 2018, our Institute launched the Diagnostic Assessment Program (DAP)

for prostate cancer. It enabled quick access to a urologist for patients presented to family physician with elevated PSA and allowed fast multidisciplinary patient care. We aim to document our data over 2 years in comparison to data before implementation of DAP and its impact on the degree of adherence to Canadian guidelines.

Methods: From April 2016 to April 2020, 880 patients who were evaluated for prostate cancer at Thunder Bay Regional Health Sciences Centre (TBRHSC) were included in this study. Patients' characteristics, clinical data, waiting times and line of treatment before and after implementation of DAP were calculated and statistically analysed.

Results: The median waiting time to urology consultation was significantly reduced from 68 (IQR 27-168) days to 34 (23-44) days $(p<0.001)$. The time from patient's referral to prostate biopsy decreased substantially from 34 (20-66) days to 18(11$25)$ days after DAP $(p<0.001)$. After DAP, the percentage of Gleason 6 detected prostate cancers were significantly increased $(19.7 \%$ to $30 \%)(p=0.02)$. After DAP, rate for intermediate-risk patients elected for external beam radiotherapy (from $53.5 \%$ to $57.9 \%, p=0.53$ ) and radical prostatectomy (from $34.5 \%$ to $39.4 \%, p=0.47$ ) increased. More compliance to Canadian guidelines was observed in intermediate risk patients (88\% vs $97.3 \%, p=.008)$.

Conclusions: Implementation of DAP has led to a notable reduction of waiting time to urology consult and prostate biopsy.

There is significant increase in Gleason 6 detected prostate cancer. Increased compliance to Canadian guidelines was detected in intermediate risk patients.
\end{abstract}

KEY WORDS: Prostate cancer; Diagnostic assessment program; Prostate biopsy.

Submitted 21 September 2021; Accepted 17 October 2021

\section{INTRODUCTION}

Prostate cancer (PCa) is the second commonly diagnosed malignancy in men worldwide (1). The diversity in treatment options among different risk groups of prostate cancer necessitate cooperation amongst different specialities and substantial patients' involvement (2). It is important that patients diagnosed with prostate cancer get assessed promptly, preferably in multidisciplinary clinics (MDC) that are composed of radiation oncologists, urologist and other assisting specialists like medical oncologists, radiologists, and pathologists (3).

Analysis of many MDCs versus standard community care consistently showed that MDCs were associated with "changes in staging/diagnosis, initial management plans, higher rates of treatment, shorter time to treatment after diagnosis, better survival, and adherence to clinical guidelines" (4). Patient satisfaction and feeling of well-being was also increased due to the patient feeling well informed in treatment decisions (5). The MDC approach allows multiple specialists contribute to the treatment decisions, which has been shown to remove physician bias toward the modality of treatment provided (4).

The enthusiasm for unifying the referral process of prostate cancer was developed due to tendency of urologist and radiation oncologist who received the primary referral to suggest therapy that they offer (6). In 2007, a Diagnostic Assessment Program (DAP) for prostate cancer was developed in North York General Hospital and further was mandated by Cancer care Ontario for lung, colorectal and prostate cancers. The goal act of DAP is to improve timely access to care for prostate cancer patients. Early evidence showed that the DAP reduced wait times from cancer suspicion to radiotherapy by on average 2 months compared to standard community practice (7).

In the efforts to reduce waiting time, multiparametric magnetic resonant imaging (mpMRI) is strongly recommended in men candidate for prostate biopsy or in men enrolled in active surveillance protocols (8).

In a recent report, the surveyed physician reported less than 4 weeks waiting time to get mpMRI with further acceleration of diagnostic process (9).

A DAP has been established in Thunder Bay, Ontario since 2018. It is not clear if data collected from the DAP in Southern Ontario can be extrapolated to a more rural and remote location with a different population, less healthcare resources, and vast geographical coverage.

In a recent report on the influence of socioeconomic and geographical factors on prostate cancer diagnosis, only $17 \%$ of patients presented with localised prostate cancer live in rural area less than 4000 inhabitants (10).

We herein report the results of a retrospective analysis of the referral process before and after the implementation of the DAP, as well as the adherence to the guidelines.

No conflict of interest declared. 


\section{Patient and methods}

Electronic medical records for patients referred with suspected diagnosis of prostate cancer to our institute between 2016 and 2020 were reviewed and enrolled in this retrospective study after obtaining ethical board committee approval.

In 2018, Diagnostic Assessment Program (DAP) for prostate cancer was implemented in our institute where any patient referred with elevated PSA and/or suspicious digital rectal exam was briefly evaluated by the DAP coordinator. A structured referral form for each patient was created includin patient demographics, PSA, digital rectal exam, family history of prostate cancer, and other available clinical data. Expediated approach was used for the evaluation of the patients in specialized DAP clinic. All patients were evaluated by a urologist who discussed the management plan. For patients diagnosed with prostate cancer, all clinical data were discussed in our multidisciplinary genitourinary oncology weekly meeting.

Finally, these patients get two separate meetings with both the urologist and the radiation oncologist before making a treatment decision (Figure 1).

\section{Patient cohort}

The initial registry included all patients referred with suspected diagnosis of prostate cancer. Patients with prior diagnosis of prostate cancer were excluded from the study. Patients were stratified into 2 groups according to the date of referral. Group I included patient referred to our institute before May 2018 while group II included patient referred after that.

Data obtained included patient's age, referral date, referral reason, PSA level, date of biopsy, tumor stage, Gleason Score (GS), percent core involvement, and treatment deci- sion. Patients were classified into three risk groups according to the D'Amico criteria (11).

For elaborating the effect of DAP implementation, patients' variables and designated treatment options were compared before and after DAP configuration in the two study groups. Moreover, within each risk group, the chosen treatment was compared with the bench-mark recommendation of the Canadian guidelines.

\section{Statistical analysis}

Categorical variables were presented through numbers and percentages, and compared between groups using Fisher's exact test. The median and interquartile range (IQR) were calculated and compared using the MannWhitney test. Patients' data was analysed using SPSS version 26 (IBM Corp., Armonk, NY). Statistical significance was defined as a two-tailed p-value less than 0.05.

\section{RESULTS}

Over the four years of the study, a total of 570 patients were included in the study. One hundred sixty-eight patients were investigated for suspicion of prostate cancer before DAP implementation and 402 of them were referred after DAP initiation and allocated to the post DAP group. Thirty-one patients had negative biopsy in the pre-DAP group while 107 patients were negative in the post-DAP group. The median age of patients in the pre-DAP and post-DAP groups was 67 and 71 years, respectively $(p=0.14)$. The distribution of clinicopathological data per group were presented in Table 1.

By comparing the two study groups it was shown that, median waiting time for receiving urology consultation and prostate biopsy were substantially reduced (68 to

34 days and 34 to 18 days respectively, $\mathrm{p}<0.001$ ).

Additionally, the proportion of patients who had a negative prostatic biopsy increased significantly ( $\mathrm{p}=0.03$ ). The percentage of Gleason 6 detected prostate adenocarcinoma was increased $(19.7 \%$ vs $30.5 \%, \mathrm{p}=$ 0.02 ) while Gleason 7 detected one were significantly decreased (50\% vs $29.6 \%, \mathrm{p}=0.002$ ).

After DAP, rate of intermediaterisk patients elected for external beam radiotherapy (from 53.5\% to $57.9 \%, \mathrm{p}=0.53)$ and radical prostatectomy (from $34.5 \%$ to $39.4 \%, \mathrm{p}=0.47)$ increased. 
Table 1.

Comparison of frequency of demographic variables and potential risk factors in each study group.

\begin{tabular}{|l|c|c|c|}
\hline Demographic & $\begin{array}{c}\text { Pre-DAP group } \\
\mathbf{n = 1 6 8}\end{array}$ & $\begin{array}{c}\text { Post-DAP Group } \\
\mathbf{n}=\mathbf{4 0 2}\end{array}$ & P-value \\
\hline Age years & $67(69-63)$ & $71(72-69)$ & 0.14 \\
\hline Median (IQRZ-IQR1) & $7.3(7.6-6.5)$ & $6.9(7.1-6.4)$ & 0.08 \\
\hline $\begin{array}{l}\text { Serum PSA level (mg/dL) } \\
\text { Median (IQR3-IQR1) }\end{array}$ & $68(168-27)$ & $34(44-23)$ & $<0.001$ \\
\hline Waiting time for urology consultation (days) & $34(66-20)$ & $18(25-11)$ & $<0.001$ \\
\hline Median (IQR3-IQR1) & $4(6-4)$ & $4(7-4)$ & 0.23 \\
\hline Waiting time for prostate biopsy (days) & $30(50-25)$ & $35(45-30)$ & 0.27 \\
Median (IQR3-IQR1) & $31(18.4 \%)$ & $107(26.6 \%)$ & 0.03 \\
\hline Number of positive prostate biopsy core & $33(19.7 \%)$ & $123(30.5 \%)$ & 0.02 \\
Median (IQR3-IQR1) & $84(50 \%)$ & $119(29.6 \%)$ & 0.001 \\
\hline Percentage of cancer involvement & $20(11.7 \%)$ & $53(13.2 \%)$ & 0.1 \\
Median (IQR3-IQR1) & & & \\
\hline Negative biopsy & & & \\
Gleason 6 & & & \\
Gleason 7 & & & \\
Gleason > 8 & & & \\
\hline
\end{tabular}

Following DAP, 97.3\% of intermediate-risk patients received a treatment according to Canadian guidelines $1^{\text {st }}$ line recommendation which was significantly higher than the rate prior to DAP ( $88 \%$ vs $97.3 \%, \mathrm{p}=0.008$ ) (Figure 2, Table 2).

Table 2.

Primary treatment for intermediate risk category.

\begin{tabular}{|l|c|c|c|}
\hline Treatment options & $\begin{array}{c}\text { Pre-DAP } \\
\mathbf{n = 8 4}\end{array}$ & $\begin{array}{c}\text { Post-DAP } \\
\mathbf{n = 1 1 9}\end{array}$ & P-value \\
\hline Active surveillance $(\mathrm{n})$ & $6(7.1 \%)$ & 0 & - \\
\hline Radical prostatectomy $(\mathrm{n})$ & $29(34.5 \%)$ & $47(39.5 \%)$ & 0.47 \\
\hline Radiation therapy (n) & $45(53.5 \%)$ & $69(57.9 \%)$ & 0.53 \\
\hline Hormonal therapy (n) & $4(4.7 \%)$ & $3(2.5 \%)$ & 0.7 \\
\hline
\end{tabular}

\section{Figure 2.}

Distribution of treatment choice for intermediate risk group.

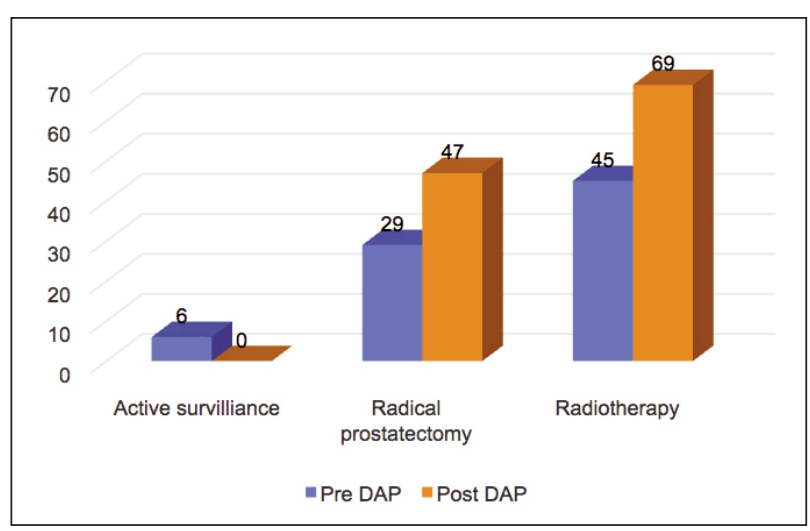

\section{Discussion}

A centralised, organised system with a multidisciplinary approach is critical for expediting the delivery of diagnostic cancer assessment services (12). The diagnostic assessment program is an evidence-based approach that originates from published literature, environmental scan and the opinion of related expertise who reach to a consensus on the standard organized diagnostic assessment services in Ontario (13).

One of the critical issues in approaching the prostate cancer cases is the time gap between diagnosis and the provided treatment. According to a prospective Canadian study, the median of waiting time for prostate cancer diagnosis was about 81 days (14). In an Irish prospective study evaluating rapid access diagnostic clinic, the median waiting time from referral date to urology consultation was 13 days (range, 1-37) (15). The Calgary Prostate Institute's rapid access clinic (RAC) reported a median wait time of 21 days from referral by primary care provider to prostate biopsyv (16). In our series, we reported 34 days median waiting time from referral to urology consultation.

The differences in the median waiting time in the Irish (15), Calgary Institute (16) and our study may be attributed to the differences in catchment areas and the unique geographical characteristic of Northern Ontario. The area of coverage of Northwestern Ontario goes up to 526.000 $\mathrm{Km}^{2}$, with numerous remote reserves and smaller towns. The changes of Gleason grade detection in relation to changes in referral pathway have been previously studies. Gilliland et al. described Gleason grade migration in response to change in detection method from incidental finding to screening (17). In a comparative study evaluating prostate cancer rapid access diagnostic clinic, O'Kelly et al. reported a downward migration in Gleason grades with significant increase in Gleason 6 detected prostate cancer (51\% vs 18\%) (18). Guy et al., on the other hand, reported decrease in diagnosing low risk disease and increase in intermediate risk disease after initiation of multidisciplinary diagnostic assessment programme (5). We also identified increase in low-risk prostate cancer. These differences in results may be due to variability in studies design and discrepancies between multidisciplinary approach or diagnostic assessment program. Additionally, we think that the facilitation of the referral process has led to an increase in the number of referrals which might have caused the increase in the number of cases with low-risk prostate cancer. The higher grade prior to DAP can also be attributed to the effect of the US task force recommendation $(19,20)$.

There is a growing interest in literature to link the multidisciplinary approach for prostate cancer management and the degree of care patients received and guidelines adherence. In a study of 630 patients from 3 tertiary care centers, Aizer et. al. reported that patients managed through multidisciplinary approach were opted to active surveillance more than patients managed by a single speciality (64\% vs $30 \%$; $\mathrm{p}<0.001)(21)$. The investigators concluded that multidisciplinary approach would lead to more adherence to National Comprehensive Cancer Network (NCCN) guidelines for very low risk prostate cancer and avoidance of unnecessary treatments. In our study, we aimed to look at the intermediate risk group as the options are clearer with fewer variabilities compared to the guideline's recommendation for the lower or higher risk groups. Another group of investigators noted significant adherence to NCCN guidelines compared to the period before initiation of multidisciplinary clinic in intermediate risk group (89.8\% vs. $76 \%, p=0.01$ ), while it was not statistically significant in 
low-risk $(100 \%$ vs. $99 \%, p=0.43)$ and high-risk patients (100\% vs. $95 \%, p=0.26)$ (22). Similarly, our results showed significant adherence to Canadian guidelines in the intermediate risk group $(\mathrm{p}=0.008)$.

Our study is not void of limitations. Firstly, the retrospective nature of our study is considered a design limitation. Secondly, all participants in this research were seen in our tertiary care facility, a context that facilitates the implementation of multidisciplinary clinics more easily than community hospitals with geographical restrictions. Lastly, our data analysis is limited to 4 years period and a longer time would warrant more accurate results.

\section{Conclusions}

Implementation of DAP has led to a notable reduction of waiting time to urology consult and prostate biopsy. There is significant increase in Gleason 6 detected prostate cancer. Increased compliance to Canadian guidelines was detected in intermediate risk patients.

\section{REFERENCES}

1. D'Agostino D, Corsi P, Colicchia M, et al. The pathological and clinical features of anterior lesions of prostate cancer: evaluation in a single cohort of patients. Arch Ital Urol Androl. 2020; 92:102

2. Horwich A, Hugosson J, de Reijke T, et al. Prostate cancer: ESMO consensus conference guidelines 2012. Ann Oncol. 2013; 24:1141-62.

3. Valicenti RK, Gomella L, El-Gabry E, et al. The multidisciplinary clinic approach to prostate cancer counseling and treatment. Semin. Urol. Oncol. 2000; 18:188-191.

4. Pillay B, Wootten AC, Crowe H, et al. The impact of multidisciplinary team meetings on patient assessment, management and outcomes in oncology settings: A systematic review of the literature. Cancer Treat Rev. 2016; 42:56-72

5. Guy D, Ghanem G, Loblaw A, et al. Diagnosis, referral, and primary treatment decisions in newly diagnosed prostate cancer patients in a multidisciplinary diagnostic assessment program. Can Urol Assoc J. 2016; 10:120.

6. Keyes M, Crook J, Morris WJ, et al. Canadian prostate brachytherapy in 2012. Can Urol Assoc J. 2013; 7:51-8

7. Sethukavalan $P$, Zhang L, Jethava $V$, et al. Improved wait time intervals for prostate cancer patients in a multi-disciplinary rapid diagnostic unit compared to a community-based referral pattern. Can Urol Assoc J. 2013; 7:244.

8. Pepe P, Candiano G, Pepe L, et al. mpMRI PI-RADS score 3 lesions diagnosed by reference vs affiliated radiological centers: Our experience in 950 cases. Arch Ital Urol Androl. 2021; 93:139-142.

9. Stanzione A, Creta M, Imbriaco M, et al. Attitudes and perceptions towards multiparametric magnetic resonance imaging of the prostate: A national survey among Italian urologists. Arch Ital Urol Androl. 2020; 9:291.

10. Pereira-Lourenço M, Vieira E Brito D, Peralta JP, et al. Influence of sociodemographic factors on treatment's choice for localized prostate cancer in Portugal. Arch Ital Urol Androl. 2020; 92:45-49.

11. D'Amico AV, Whittington $R$, Schultz D, et al. Outcome based staging for clinically localized adenocarcinoma of the prostate. J Urol. 1997; 158:1422-1426.

12. Brouwers $M$, Crawford J, Elison P, et al. Organizational standards for diagnostic assessment programs. Toronto $(\mathrm{ON})$ : Cancer
Care Ontario; 2007 Jun 15 (In review 2011 Sep). Program in Evidence-based Care Evidence-based Series Organizational Standards for DAP IN REVIEW

13. Brouwers M, Oliver TK, Crawford J, et al. Cancer diagnostic assessment programs: standards for the organization of care in Ontario. Curr Oncol. 2009; 16:29-41.

14. Grunfeld E, Watters JM, Urquhart R, et al. A prospective study of peri-diagnostic and surgical wait times for patients with presumptive colorectal, lung, or prostate cancer. Br J Cancer. 2009; 100:56-62.

15. Forde JC, O'Connor KM, Casey L, et al. A rapid access diagnostic clinic for prostate cancer: the experience after one year. Ir J Med Sci. 2011; 180:505-8.

16. Kavanagh AG, Lee JC, Donnelly B. Time to treatment of prostate cancer through the Calgary Prostate Institute rapid access clinic. Can J Urol. 2008; 15:3975-3979.

17. Gilliland FD, Gleason DF, Hunt WC, et al. Trends in Gleason score for prostate cancer diagnosed between 1983 and 1993. J Urol. 2001; 165:846-850

18. O'Kelly F, Thomas AZ, Murray D, et al. Emerging evidence for Gleason grade migration and distance impact in prostate cancer? An analysis of the rapid access prostate clinic in a tertiary referral center: St. Vincent's University Hospital, Dublin (2009-2011). Ir J Med Sci. 2013; 182:487-91.

19. Moyer VA,US Preventive Services Task Force. Screening for prostate cancer: U.S. Preventive Services Task Force recommendation statement. Ann Int Med. 2012; 157:120-134.

20. Butler SS, Muralidhar V, Zhao SG, et al. Prostate cancer incidence across stage, NCCN risk groups, and age before and after USPSTF Grade D recommendations against prostate-specific antigen screening in 2012. Cancer. 2020; 126:717-724.

21. Aizer AA, Paly JJ, Zietman AL, et al. Models of care and NCCN guideline adherence in very-low-risk prostate cancer. J Natl Compr Canc Netw 2013; 11:1364-72.

22. Korman H, Lanni TJr, Shah C, et al. Impact of a prostate multidisciplinary clinic program on patient treatment decisions and on adherence to NCCN guidelines: the William Beaumont Hospital experience. Am J Clin Oncol. 2013; 36:121-125.

\section{Correspondence}

Walid Shabana, MD (Corresponding Author)

waleed.shabana@gmail.com

Ahmed Kotb, MD

kotba@tbh.net

Daniel Tesolin, MD

dtesoln@nosm.ca

Mohammed Ibrahim, MD

ibrahimm@tbh.net

Kristi Dolcetti, MD

dolcetk@tbh.net

Amy Boucher, MD

bouchra@tbh.net

Mohammed Bassuony, MD

Bassunm@tbh.net

Kevin Ramchandar, MD

ramchnk@tbh.net

Ahmed Zakaria, MD

aszakari81@yahoo.com

Hazem Elmansy, MD

elmancyh@tbh.net

Walid Shahrour, MD

walid.shahrou@gmail.com

Urology Department, Northern Ontario School of medicine,

146 Court Street South, Thunder Bay, ON P7B 2X6, Canada 